\title{
Symptomatic, prolonged Parvovirus B19 infection and accompanying illness in otherwise healthy adults
}

Roberto Manfredi

From $16^{\text {th }}$ International Symposium on HIV and Emerging Infectious Diseases

Marseille, France. 24-26 March 2010

\section{Background}

Parvoviridae are part of air-, parenteral- and perinataltransmitted ubiquitous viruses, whose associated signs and symptoms strongly depend on patient's age and immune defence.

\section{Methods}

All cases of symptomatic Parvovirus B19 infection in otherwise healthy adults which came to our attention since spring 2006 were prospectively investigated and followed-up.

\section{Results}

In a 21-month period, 11 patients ( 7 females and 4 males), with a mean age of 36.9 (range 27-46) years with a symptomatic Parvovirus B19 infection were recorded and followed-up. Intrafamiliar exposure and occupational (health care) exposure were identified in two cases each. Clinical signs and symptoms included fever (100\% of cases), polyarthralgia (90\%), followed by headache $(80 \%)$, anemia $(70 \%)$, and rash. A mild-tomoderate myelosuppression of all hematological lines characterized 8 cases of 11 (72.7\%), while increased serum transaminases were associated in $63.6 \%$ of patients. Three patients of ours deserved hospitalization (mean 10.8 days of admission), and five more cases were followed on Day-Hospital basis (for a median 75-day period); in four patients a treatment with i.v. highdosage human immunoglobulins was performed. Elevated levels of specific serum anti-Parvovirus B19 IgM antibodies were detected in all cases. In a 33-year-old female a severe anemia and a persisting headache, vomiting, and neck stiffness, led to $\mathrm{RBC}$ transfusion and a diagnosis of meningoencephalitis, with positive search of IgM antibodies and Parvovirus B19 viremia (detected by RT-PCR) in the cerebrospional fluid which lasted up to three months, despite treatment with i.v. serum immunoglobulins.

\section{Discussion}

Parvovirus B19 infection may play a significant role also in adult, immunocompetent subjects, and the disease sometimes is not mild and self-limiting, requiring admission and/or frequent outpatient interventions in a significant number of cases. The causes supporting a persistant infection in immunocompetent subjects have not been investigated to date, as well as the pathogenesis of myelosuppression and severe polyartrhalgia. Symptomatic Parvovirus B19 infection is still an underestimated clinical condition, and therapeutic perspectives are extremely limited until now.

Published: 11 May 2010

doi:10.1186/1742-4690-7-S1-P179

Cite this article as: Manfredi: Symptomatic, prolonged Parvovirus B19 infection and accompanying illness in otherwise healthy adults. Retrovirology 2010 7(Suppl 1):P179.

Correspondence: Roberto.manfredi@unibo.it

Infectious Diseases, University of Bologna, Bologna, Italy 